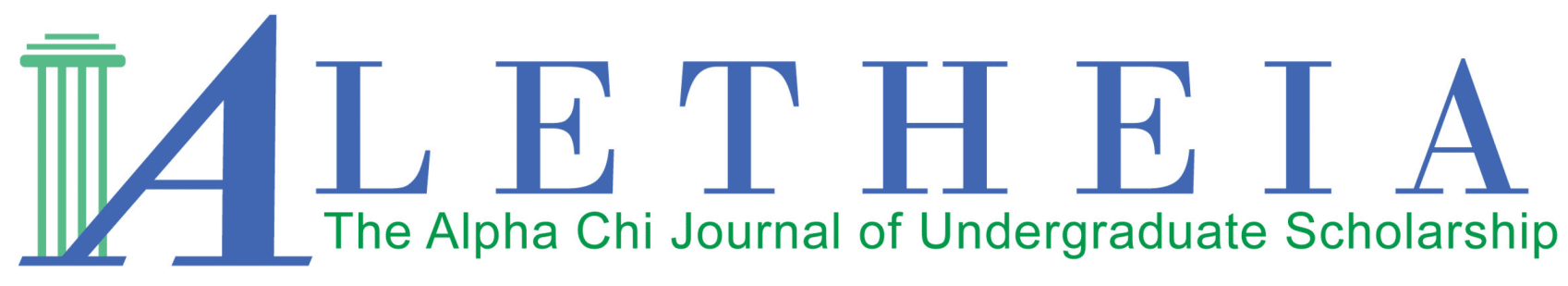

Volume 2 | Issue 1 | 2017

\title{
Circles in $\mathbb{F}_{q}^{2}$
}

\author{
Jacob Haddock; Wesley Perkins; John Pope; and \\ Jeremy Chapman, $\mathrm{PhD}$
}

Lyon College

Arkansas Iota Chapter

Vol. 2(1) 2017

Article Title: Circles in $\mathbb{F}_{q}^{2}$

DOI: $10.21081 / \mathrm{ax} 0085$

ISSN: $2381-800 \mathrm{X}$

Key Words: Finite fields, circles.

This work is licensed under a Creative Commons Attribution 4.0 International License.

Author contact information is available from the Editor at editor@alphachihonor.org.

\section{Aletheia-The Alpha Chi Journal of Undergraduate Scholarship}

- This publication is an online, peer-reviewed, interdisciplinary undergraduate journal, whose mission is to promote high quality research and scholarship among undergraduates by showcasing exemplary work.

- Submissions can be in any basic or applied field of study, including the physical and life sciences, the social sciences, the humanities, education, engineering, and the arts.

- Publication in Aletheia will recognize students who excel academically and foster mentor/mentee relationships between faculty and students.

- In keeping with the strong tradition of student involvement in all levels of Alpha Chi, the journal will also provide a forum for students to become actively involved in the writing, peer review, and publication process.

- More information and instructions for authors is available under the publications tab at www.AlphaChiHonor.org. Questions to the editor may be directed to editor@alphachihonor.org.

Alpha Chi is a national college honor society that admits students from all academic disciplines, with membership limited to the top 10 percent of an institution's juniors, seniors, and graduate students. Invitation to membership comes only through an institutional chapter. A college seeking a chapter must grant baccalaureate degrees and be regionally accredited. Some 300 chapters, located in almost every state, induct approximately 12,000 members annually. Alpha Chi members have been "making scholarship effective for good" since 1922. 


\title{
Circles in $\mathbb{F}_{q}^{2}$
}

\author{
Jacob Haddock; Wesley Perkins; John Pope; and Jeremy Chapman, PhD \\ Lyon College \\ Arkansas Iota Chapter
}

\begin{abstract}
In Euclid's The Elements, a unique circle in $\mathbb{R}^{2}$ is determined by three noncollinear points. This is proven geometrically by constructing a triangle from the three points and showing that the intersection of the perpendicular bisectors of two sides of the triangle gives a point that is equidistant from all three vertices of the triangle [2]. This point is said to define the center of a circle which circumscribes the triangle formed by the points. In our research, we demonstrate that circles can be similarly determined in $\mathbb{F}_{q}^{2}$, the two-dimensional vector space over the finite field $\mathbb{F}_{q}$. However, the properties of $\mathbb{F}_{q}^{2}$ cause some interesting cases to arise. Among these is the possibility for two distinct points to have zero distance. Nevertheless, we show that three distinct noncollinear points which have nonzero distance from each other determine a unique circle of nonzero radius.
\end{abstract}

Keywords: Finite fields, circles

\section{Introduction}

In our project, work was done specifically in $\mathbb{F}_{q}^{2}$. For completeness, we define the following:

Definition 1.1. The set $G$ defines a group with respect to the binary operation $*$ if the following are satisfied:

1. $G$ is closed under $*$.

2. $*$ is associative.

3. $G$ has an identity element, $e$.

4. Every element of $G$ has an inverse. For each $a \in G$, there exists $b \in G$ such that $a * b=b * a=e$.

Note that if * on $G$ is commutative, then $G$ is called an abelian group.

Definition 1.2. The set $R$ defines a ring with respect to addition and multiplication if the following are satisfied:

1. $R$ forms an abelian group with respect to addition.

2. $R$ is closed with respect to an associative multiplication.

3. The following two distributive laws hold: $x(y+z)=$ $x y+x z$ and $(x+y) z=x z+y z$.
Note that if multiplication in $R$ is commutative, then $R$ is called a commutative ring.

Definition 1.3. The set $F$ defines a field if the following are satisfied:

1. $F$ is a commutative ring.

2. $F$ has a unity $1 \neq 0$ such that $1 \cdot x=x \cdot 1=x$ for all $x \in F$.

3. Every nonzero element of $F$ has a multiplicative inverse.

Every field is an integral domain, meaning that there are no zero divisors [3]. Zero divisors are nonzero elements of the integral domain, say $a \neq 0$, which can be multiplied by another nonzero element, say $b \neq 0$, to yield zero: $a b=0$.

Definition 1.4. A field that has a finite number of elements is called a finite field. It is known that the order of every finite field is the power of a prime [4]. In this paper, we use $\mathbb{F}_{q}$ to denote a finite field with $q$ elements where $q=p^{l}, p>2$ is a prime, and $l \in \mathbb{N}$. Note that since $\mathbb{F}_{q}$ is a finite integral domain, the characteristic of the unity 1 is $p$ [3]. In other words, $p$ is the least positive integer such that $p \cdot 1=0$. Since $p>2$ it follows that $2 \neq 0$, and we will use this fact. 
Remark 1.5. Throughout this paper, we will use the following notation:

1. $\frac{a}{b}$ will represent $(a)\left(b^{-1}\right)$, where $b^{-1}$ is the multiplicative inverse of $b$.

2. $a-b$ will represent $a+(-b)$, where $-b$ is the additive inverse of $b$.

Definition 1.6. Let $\mathbb{F}$ be a field. A vector space is a set $V$ along with an addition on $V$ and a scalar multiplication on $V$ such that the following properties hold (see [1]):

1. $u+v=v+u$ for all $u, v \in V$.

2. $(u+v)+w=u+(v+w)$ and $(a b) v=a(b v)$ for all $u, v, w \in V$ and all $a, b \in \mathbb{F}$.

3. There exists an element $0 \in V$ such that $v+0=v$ for all $v \in V$.

4. For every $v \in V$, there exists $w \in V$ such that $v+w=0$.

5. $1 v=v$ for all $v \in V$.

6. $a(u+v)=a u+a v$ and $(a+b) u=a u+b u$ for all $a, b \in \mathbb{F}$ and all $u, v \in V$.

Definition 1.7. In this paper we work exclusively in $\mathbb{F}_{q}^{2}$ the two-dimensional vector space over the finite field $\mathbb{F}_{q}$. Just as $\mathbb{R}^{2}$ is the set of all ordered pairs of real numbers, one can think of $\mathbb{F}_{q}^{2}$ as all ordered pairs of elements of $\mathbb{F}_{q}$, that is, $\mathbb{F}_{q}^{2}=\left\{(x, y): x, y \in \mathbb{F}_{q}\right\}$.

Definition 1.8. The norm, or distance, between two points $P_{1}=\left(x_{1}, y_{1}\right)$ and $P_{2}=\left(x_{2}, y_{2}\right)$ where $P_{1}, P_{2} \in \mathbb{F}_{q}^{2}$, denoted $\left\|P_{2}-P_{1}\right\|$, is $\left(x_{2}-x_{1}\right)^{2}+\left(y_{2}-y_{1}\right)^{2}$.

Note that, because we are working in $\mathbb{F}_{q}^{2}$, it is possible for two distinct points to possess zero distance.

Example 1.9. Consider $\mathbb{Z}_{5}^{2}$, the two-dimensional vector space over the finite field $\mathbb{Z}_{5}$. We use bar notation to denote the congruence classes, that is, $\mathbb{Z}_{5}=\{\overline{0}, \overline{1}, \overline{2}, \overline{3}, \overline{4}\}$. In this particular field, modular arithmetic allows us to demonstrate zero distance between the points $(\overline{2}, \overline{1})$ and $(\overline{0}, \overline{0})$. Substituting these points into the norm equation, we get $\left\|P_{2}-P_{1}\right\|=(\overline{2}-\overline{0})^{2}+(\overline{1}-\overline{0})^{2}=\overline{4}+\overline{1}=\overline{5}=\overline{0}$, since $5 \equiv 0(\bmod 5)$.

Later, we will utilize more interesting consequences of the zero norm problem.

Definition 1.10. Let $\mathbb{F}_{q}[x]$ denote the polynomial ring over $\mathbb{F}_{q}$. Let $f \in \mathbb{F}_{q}[x]$ be a polynomial of degree one, that is, $f(x)=m x+b$ where $m, b \in \mathbb{F}_{q}$. We define a nonvertical line in $\mathbb{F}_{q}^{2}$ to be the set $\left\{\left(x_{0}, y_{0}\right) \in \mathbb{F}_{q}^{2}: y_{0}=m x_{0}+b\right\}$. We say that $m$ is the slope and $(0, b)$ is the y-intercept. For $a \in \mathbb{F}_{q}$, the vertical line $x=a$ is defined to be the set $\left\{(a, y): y \in \mathbb{F}_{q}\right\}$.
Definition 1.11. The perpendicular bisector of the line segment $\overline{P_{1} P_{2}}$, denoted bisector $\left(P_{1}, P_{2}\right)$, is given by:

$$
\text { bisector }\left(P_{1}, P_{2}\right)=\left\{P \in \mathbb{F}_{q}^{2}:\left\|P_{1}-P\right\|=\left\|P_{2}-P\right\|\right\} \text {. }
$$

Note that the perpendicular bisector is a line and the slope is still the negative reciprocal of the line it bisects. This will be demonstrated in Section 3.1.

Definition 1.12. We shall refer to the perpendicular bisector of two points that are zero norm from one another as a zero line. We will show in Lemma 2.2 that the zero line is precisely the line through the two points.

Definition 1.13. A circle is defined as the set of all points equidistant from an arbitrary center. In particular, a circle centered at $C$ of radius $r$ is given by $S_{r}(C)=$ $\left\{P \in \mathbb{F}_{q}^{2}:\|C-P\|=r\right\}$.

Figure 1 displays a circle of radius zero over $\mathbb{Z}_{5}^{2}$ to give an example of what such a circle would look like. Figure 2 demonstrates a circle of nonzero radius over $\mathbb{Z}_{7}^{2}$.

Theorem 1.14. Let $P_{1}, P_{2}, P_{3} \in \mathbb{F}_{q}^{2}$ be three distinct, noncollinear points that are nonzero norm from each other. Then, these points determine a unique circle of nonzero radius in $\mathbb{F}_{q}^{2}$.

In order to prove Theorem 1.14, we must first prove several lemmas which allow us to justify the nonzero claims of the theorem. The first lemma demonstrates that, when $\left\|P_{2}-P_{1}\right\|=0$, the perpendicular bisector of $\overline{P_{1} P_{2}}$ is the line containing points $P_{1}, P_{2} \in \mathbb{F}_{q}^{2}$. The second lemma uses the first to demonstrate that an arbitrary point $P \in$ $\mathbb{F}_{q}^{2}$ is zero norm from $P_{1}$ and $P_{2}$ if it lies on the perpendicular bisector of $\overline{P_{1} P_{2}}$ and $\left\|P_{2}-P_{1}\right\|=0$. The third lemma demonstrates that if the square root of an element of $\mathbb{F}_{q}$ exists, then there are exactly two square roots that exist so long as the element is not zero. The fourth lemma uses the third to demonstrate that if an arbitrary point $P \in \mathbb{F}_{q}^{2}$ lies on a zero line, then it has exactly two zero lines that pass through it. The fifth lemma uses the second and fourth to demonstrate that if $P_{1}, P_{2}$, and $P_{3}$ are nonzero norm from one another and are noncollinear, then their perpendicular bisectors do not intersect at a point that is zero norm from $P_{1}, P_{2}$, and $P_{3}$, thus ruling out circles of radius zero.

We exclude circles of zero radius on the basis that the unique properties of $\mathbb{F}_{q}^{2}$ are expected to alter the behavior of circles to such a degree that it warrants a separate in-depth investigation. Our proof of Theorem 1.14 is a direct proof which uses Definition 1.11 to algebraically derive expressions for the center of the circle defined by three distinct, noncollinear points, the existence of which is verified by satisfying Definition 1.13. This proof also requires us to verify that the center exists and is unique, and validate any division operations required to reach our conclusion. 


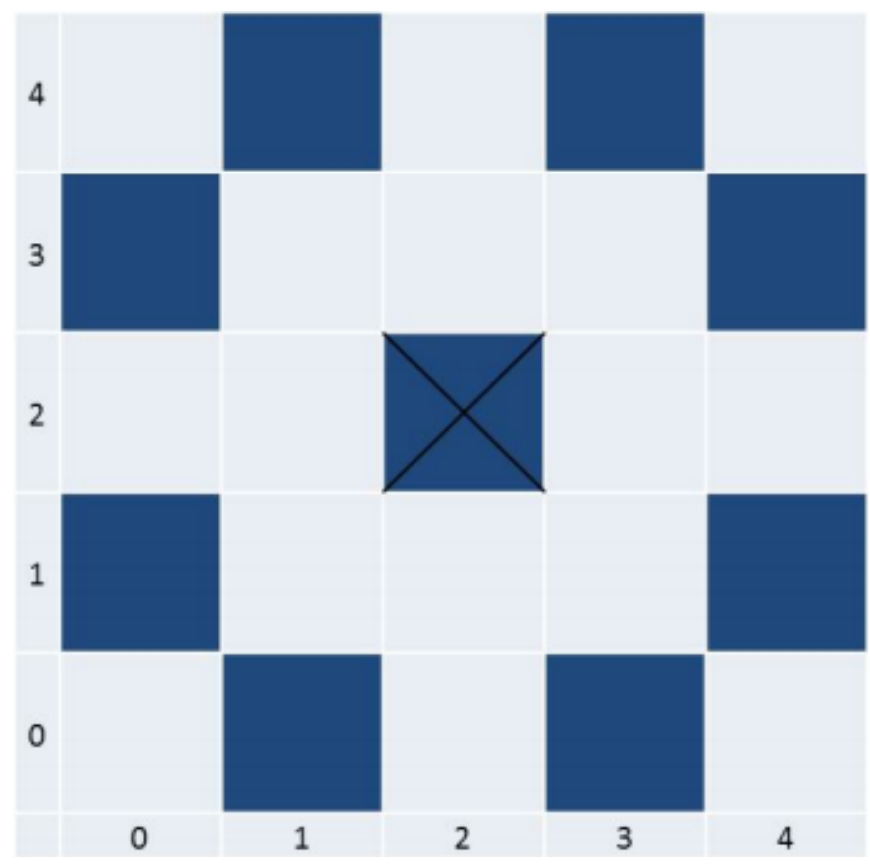

Figure 1: A circle of radius zero centered at $(\overline{2}, \overline{2})$ over $\mathbb{Z}_{5}^{2}$. It is worth noting that in the case of a zero radius circle, the center of the circle is actually included as part of the circle.

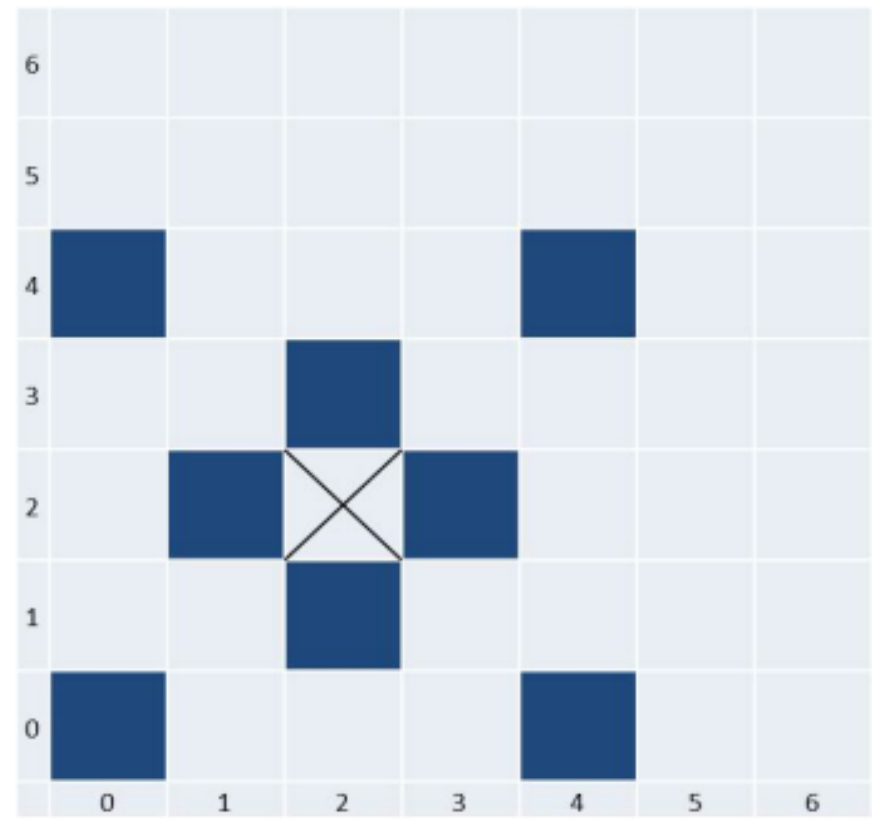

Figure 2: A circle of radius one centered at $(\overline{2}, \overline{2})$ over $\mathbb{Z}_{7}^{2}$

\section{Proof of Lemmas}

Remark 2.1. Throughout the presented proofs involving zero lines, we multiply by $\left(x_{2}-x_{1}\right)^{-1}$ and $\left(y_{2}-y_{1}\right)^{-1}$. This multiplication is valid as long as $\left(x_{2}-x_{1}\right)^{-1}$ and $\left(y_{2}-y_{1}\right)^{-1}$ exist, or in other words, as long as $x_{1} \neq x_{2}$ and $y_{1} \neq y_{2}$. We can verify that this is the case by considering the following:

If $x_{2}-x_{1}=0$ and $y_{2}-y_{1}=0$, i.e. $x_{1}=x_{2}$ and $y_{1}=y_{2}$, then we have only one point, $P_{1}=P_{2}$, a violation of the initial conditions of our proofs.

If we let one set of coordinates be equal, say $x_{1}=x_{2}$, i.e. $x_{2}-x_{1}=0$, and $\left\|P_{2}-P_{1}\right\|=\left(x_{2}-x_{1}\right)^{2}+\left(y_{2}-y_{1}\right)^{2}=0$ (since we will be specifically investigating zero norms), then:

$$
\begin{aligned}
\left\|P_{2}-P_{1}\right\|=(0)^{2}+\left(y_{2}-y_{1}\right)^{2} & =0 \\
\Longrightarrow\left(y_{2}-y_{1}\right)^{2} & =0 \\
\Longrightarrow y_{2} & =y_{1} . \\
& \text { Again, implying } P_{1}=P_{2} .
\end{aligned}
$$

We get a similar result if we let $y_{1}=y_{2}$. Note that, since fields are integral domains (see Definition 1.3), it follows that the square root of zero is zero in our case. From this set of justifications, we are also guaranteed that it is impossible to have a horizontal or vertical zero line (since $x_{1} \neq x_{2}$ and $y_{1} \neq y_{2}$ for any two arbitrary points on an arbitrary zero line).

Lemma 2.2. The perpendicular bisector of two distinct points zero norm apart is the line containing the two points. Recall that we shall call such a line a zero line.

Proof. Suppose there exists two points $P_{1}, P_{2} \in \mathbb{F}_{q}^{2}$ such that $\left\|P_{2}-P_{1}\right\|=0$. To show the perpendicular bisector and the line $\stackrel{P_{1} P_{2}}{\longrightarrow}$ are the same, it suffices to show both lines contain two points in common.

To begin, we make note of the fact that, as demonstrated in 3.1, we have that the perpendicular bisector of any two points in $\mathbb{F}_{q}^{2}$ is, in fact, a line.

Now we want to show that both the line $\overleftrightarrow{P_{1} P_{2}}$ and the perpendicular bisector of $P_{1}$ and $P_{2}$ contain two points in common. To do this, consider the definition of a perpendicular bisector:

$$
\text { bisector }\left(P_{1}, P_{2}\right)=\left\{P \in \mathbb{F}_{q}^{2}:\left\|P_{1}-P\right\|=\left\|P_{2}-P\right\|\right\} .
$$

If we pick the point $P_{1}$, which is on $\overleftarrow{P_{1} P_{2}}$, it follows that $P_{1}$ is also in $\operatorname{bisector}\left(P_{1}, P_{2}\right)$, since $\left\|P_{1}-P_{1}\right\|=0$ and $\left\|P_{2}-P_{1}\right\|=0$ by assumption. This can also be shown for $P_{2}$. Since $P_{1}$ and $P_{2}$ both lie in the bisector, it follows that the bisector of $P_{1}$ and $P_{2}$ is the same line as $\overleftrightarrow{P_{1} P_{2}}$.

Lemma 2.3. An arbitrary point on the perpendicular bisector of two distinct points zero norm apart is zero norm from each of the two points.

Proof. Let $P_{1}, P_{2} \in \mathbb{F}_{q}^{2}$ be two distinct points such that $\left\|P_{2}-P_{1}\right\|=0$. Let $P_{0}$ be an arbitrary point on bisector $\left(P_{1}, P_{2}\right)$. By Lemma 2.2 we have that $\operatorname{bisector}\left(P_{1}, P_{2}\right)=\overleftrightarrow{P_{1} P_{2}}$, so we have that $P_{0}$ is a point on the line $\overleftrightarrow{P_{1} P_{2}}$. By Definition 1.11, it suffices to show $\left\|P_{0}-P_{1}\right\|=0$. 


$$
\begin{aligned}
y= & \frac{y_{2}-y_{1}}{x_{2}-x_{1}}\left(x-x_{1}\right)+y_{1} \\
\left.\quad \text { Equation for } \overleftarrow{P_{1} P_{2}}\right) & \\
y_{0}= & \frac{y_{2}-y_{1}}{x_{2}-x_{1}}\left(x_{0}-x_{1}\right)+y_{1} \\
\Longrightarrow\left\|P_{0}-P_{1}\right\|= & \left(x_{0}-x_{1}\right)^{2} \\
& +\left[\left(\frac{y_{2}-y_{1}}{x_{2}-x_{1}}\right)\left(x_{0}-x_{1}\right)+y_{1}-y_{1}\right]^{2} \\
& \quad\left(\text { Substituting } y_{0} \text { into }\left\|P_{0}-P_{1}\right\|\right) \\
\Longrightarrow\left\|P_{0}-P_{1}\right\|= & \frac{\left(x_{0}-x_{1}\right)^{2}}{\left(x_{2}-x_{1}\right)^{2}}\left[\left(x_{2}-x_{1}\right)^{2}+\left(y_{2}-y_{1}\right)^{2}\right] \\
& \left(\text { Factoring out }\left(x_{0}-x_{1}\right)^{2}\left(x_{2}-x_{1}\right)^{-2}\right) \\
\Longrightarrow\left\|P_{0}-P_{1}\right\|= & 0 \quad\left(\left\|P_{2}-P_{1}\right\|=0 \text { by assumption. }\right)
\end{aligned}
$$

Remark 2.4. Notice that Lemma 2.3 implies that any two points lying on a zero line will be norm zero apart. To see this, consider bisector $\left(P_{0}, P_{1}\right)$ which is the same zero line as $\operatorname{bisector}\left(P_{1}, P_{2}\right)$. Then Lemma 2.3 would imply that any arbitrary point is also zero norm from $P_{0}$. See Figure 3 for a specific example of this.

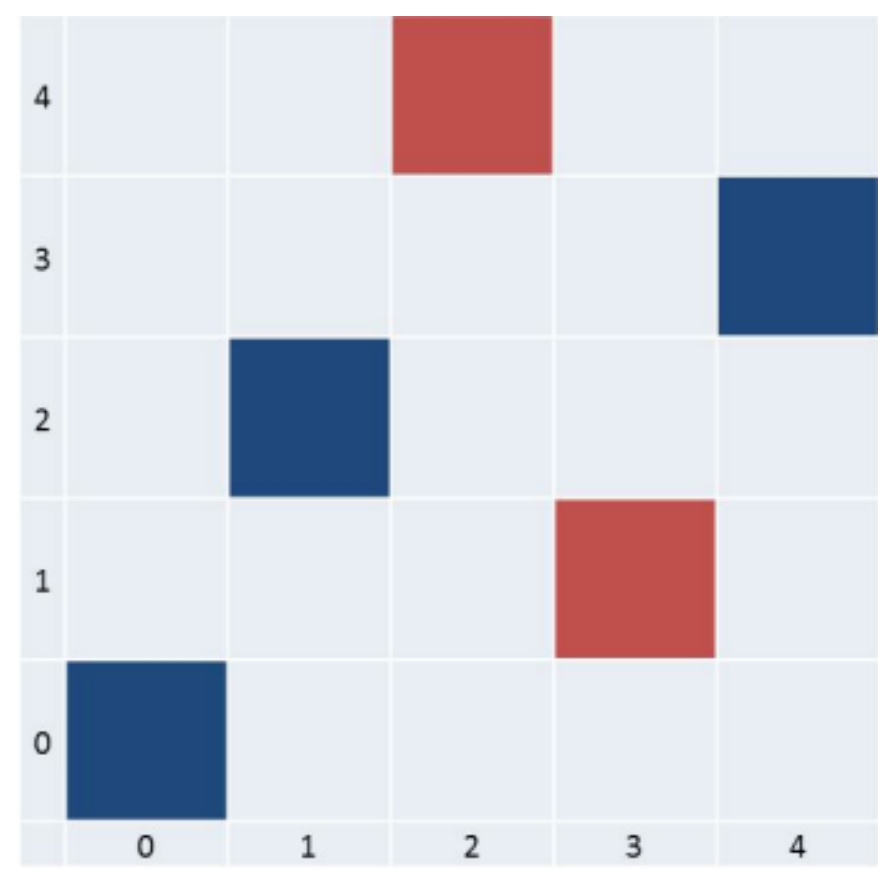

Figure 3: A zero line in $\mathbb{Z}_{5}^{2}$. Note that any two points chosen on the line will have a distance of zero from each other.
Lemma 2.5. If $a \in \mathbb{F}_{q}$ has a square root, then the equation $x^{2}=$ a has exactly two solutions as long as a is nonzero.

Proof. Suppose $a \neq 0$ has a square root, i.e. there exists $b \in \mathbb{F}_{q}$ such that $b^{2}=a$. Then,

$$
\begin{aligned}
& x^{2}=a \\
\Longrightarrow & \left.x^{2}=b^{2} \quad \text { (by substituting } a \text { with } b^{2}\right) \\
\Longrightarrow & x^{2}-b^{2}=0 \\
\Longrightarrow & (x-b)(x+b)=0 \\
\Longrightarrow & x= \pm b
\end{aligned}
$$

Here we note that since $b \neq 0$, the equation will have two solutions as long as $2 \neq 0$. Consequently, $x^{2}=a$ has exactly two solutions as long as $a$ has a square root and $2 \neq 0$. Recall $2 \neq 0$ since $p>2$.

Lemma 2.6. If an arbitrary point $P_{0}$ lies on a zero line, there are exactly two zero lines passing through $P_{0}$.

Proof. Assume that $P_{0}=\left(x_{0}, y_{0}\right) \in \mathbb{F}_{q}^{2}$ lies on a zero line. This implies that there exists $(a, b) \in \mathbb{F}_{q}^{2}$ such that $\left(x_{0}-\right.$ $a)^{2}+\left(y_{0}-b\right)^{2}=0$. Consequently, $\left(y_{0}-b\right)^{2}=-\left(x_{0}-a\right)^{2}$. In other words, $-\left(x_{0}-a\right)^{2}$ has a square root. Then, the equation $\left(y_{0}-y\right)^{2}=-\left(x_{0}-a\right)^{2}$ has solutions $y_{0}-y=$ $\pm\left(y_{0}-b\right) \Longrightarrow y=b, 2 y_{0}-b$, by Lemma 2.5. These values for $y$ are distinct because these two values can be equal only when $y_{0}=b$, which cannot happen since a zero line cannot be horizontal by Remark 2.1. Thus, there are two values for $y$, namely $y=b$ and $y=2 y_{0}-b$, that make $(a, y)$ a zero distance from $\left(x_{0}, y_{0}\right)$. Since $(a, b)$ and $\left(a, 2 y_{0}-b\right)$ are nonzero norm from each other, they must lie on separate zero lines by Remark 2.4. Lastly, it is worth mentioning that we have shown that there are exactly two zero lines through the arbitrary point $P_{0}=$ $\left(x_{0}, y_{0}\right)$. If there were a third, then there would have to be a third point $(a, y)$ such that $\left(x_{0}-a\right)^{2}+\left(y_{0}-y\right)^{2}=0$. However, we have shown above that there are only two values for $y$ that satisfy this equation.

Lemma 2.7. If three distinct points $P_{1}, P_{2}$, and $P_{3}$ are chosen such that they are nonzero norm from one another and they are noncollinear, their perpendicular bisectors cannot intersect at a point $C$ such that $\left\|P_{1}-C\right\|=\| P_{2}-$ $C\|=\| P_{3}-C \|=0$.

Proof. Let $P_{1}, P_{2}, P_{3} \in \mathbb{F}_{q}^{2}$ be points that satisfy the conditions above. Let $C$ be the unique intersection of bisector $\left(P_{1}, P_{2}\right)$ and bisector $\left(P_{2}, P_{3}\right)$. We know that the intersection is unique because bisector $\left(P_{1}, P_{2}\right)$ and bisector $\left(P_{2}, P_{3}\right)$ are nonparallel lines (see Remark 3.2). For a contradiction, consider the possibility that $\| P_{1}-$ $C\|=\| P_{2}-C\|=\| P_{3}-C \|=0$. Then $C$ shares a zero line with $P_{1}, P_{2}$, and $P_{3}$. Since, by Lemma 2.6, $C$ has only two zero lines passing through it, at least one pair 
of the points $P_{1}, P_{2}$, and $P_{3}$ must lie on the same zero line. Since all points on a zero line are zero norm from one another, this would imply that either $\left\|P_{1}-P_{2}\right\|=0$, $\left\|P_{1}-P_{3}\right\|=0$, or $\left\|P_{2}-P_{3}\right\|=0$, all of which are contradictions to the hypothesis that none of the points are zero norm from one another.

As a result of these lemmas, it follows that if we have three distinct noncollinear points and any two of the three points are norm zero from each other, then the three points determine a zero radius circle, thereby allowing us to specify a circle of nonzero radius by maintaining that the three points defining it are all nonzero norm from each other (See Theorem 1.14).

\section{Proof of Theorem 1.14}

To prove Theorem 1.14, we consider the following:

1. Three noncollinear points, $P_{1}, P_{2}, P_{3} \in \mathbb{F}_{q}^{2}$, all nonzero norm from each other.

2. The perpendicular bisectors of $\overline{P_{1} P_{2}}$ and $\overline{P_{2} P_{3}}$.

3. Some arbitrary point $C=(x, y)$, which is said to lay on each of the perpendicular bisectors at their intersection.

We want to show $C$ exists and is a unique solution for the intersection of the bisectors. This resulting solution will define the center of the circle containing $P_{1}, P_{2}$, and $P_{3}$.

\subsection{Derivation of Perpendicular Bisectors}

To obtain the bisector of $\overline{P_{1} P_{2}}$, let:

$$
\begin{gathered}
\left\|P_{1}-C\right\|=\left\|P_{2}-C\right\| \\
\quad(\text { By Definition 1.11) } \\
\Longrightarrow\left(x_{1}-x\right)^{2}+\left(y_{1}-y\right)^{2}=\left(\begin{array}{c}
\left.x_{2}-x\right)^{2}+\left(y_{2}-y\right)^{2} \\
(\text { By Definition 1.8) }
\end{array}\right. \\
\Longrightarrow x_{1}^{2}-2 x_{1} x+x^{2}+y_{1}^{2}-2 y_{1} y+y^{2} \\
=x_{2}^{2}-2 x_{2} x+x^{2}+y_{2}^{2}-2 y_{2} y+y^{2} \\
\Longrightarrow x_{1}^{2}-2 x_{1} x+y_{1}^{2}-2 y_{1} y \\
=x_{2}^{2}-2 x_{2} x+y_{2}^{2}-2 y_{2} y \\
\Longrightarrow 2 y\left(y_{1}-y_{2}\right)+2 x\left(x_{1}-x_{2}\right) \\
=x_{1}^{2}-x_{2}^{2}+y_{1}^{2}-y_{2}^{2} \\
\Longrightarrow y=-\left(\frac{x_{1}-x_{2}}{y_{1}-y_{2}}\right) x+\frac{x_{1}^{2}-x_{2}^{2}+y_{1}^{2}-y_{2}^{2}}{2\left(y_{1}-y_{2}\right)} \\
\quad\left(\text { bisector }\left(P_{1}, P_{2}\right)\right)
\end{gathered}
$$

Similarly, the bisector of $\overline{P_{2} P_{3}}$ can be obtained:

$$
\left\|P_{2}-C\right\|=\left\|P_{3}-C\right\|
$$

(By Definition 1.11)

$$
\begin{array}{r}
\Longrightarrow\left(x_{2}-x\right)^{2}+\left(y_{2}-y\right)^{2}=\left(x_{3}-x\right)^{2}+\left(y_{3}-y\right)^{2} \\
(\text { By Definition 1.8) } \\
\Longrightarrow y=-\left(\frac{x_{2}-x_{3}}{y_{2}-y_{3}}\right) x+\frac{x_{2}^{2}-x_{3}^{2}+y_{2}^{2}-y_{3}^{2}}{2\left(y_{2}-y_{3}\right)} \\
\left.\quad \text { bisector }\left(P_{2}, P_{3}\right)\right)
\end{array}
$$

Note that if either $\overline{P_{1} P_{2}}$ or $\overline{P_{2} P_{3}}$ is horizontal, then either $y_{1}-y_{2}$ or $y_{2}-y_{3}$ is zero. We must then solve for $x$ instead of $y$ to avoid dividing by zero (it is easily verified that, in such a case, the perpendicular bisector of two points, say $\left(x_{1}, y_{1}\right)$ and $\left(x_{2}, y_{2}\right)$, horizontal to one another is the vertical line $\left.x=\frac{x_{1}+x_{2}}{2}\right)$.

Because, by Definition 1.11, we know that $\left\|P_{1}-C\right\|=$ $\left\|P_{2}-C\right\|$ and $\left\|P_{2}-C\right\|=\left\|P_{3}-C\right\|$, it follows that $\left\|P_{1}-C\right\|=\left\|P_{3}-C\right\|$ and $C$ is a point equidistant from points $P_{1}, P_{2}$, and $P_{3}$; namely, the center of the circle defined by these points (see Definition 1.13). We will soon show that $C$ is unique by using the two perpendicular bisectors we derived to obtain a generalized solution for $C=(x, y)$.

\subsection{Solving for the Center}

To obtain the $x$-coordinate of $C$, let:

$$
\begin{gathered}
\text { bisector }\left(P_{1}, P_{2}\right)=\text { bisector }\left(P_{2}, P_{3}\right) \\
\Longrightarrow-\left(\frac{x_{1}-x_{2}}{y_{1}-y_{2}}\right) x+\frac{x_{1}^{2}-x_{2}^{2}+y_{1}^{2}-y_{2}^{2}}{2\left(y_{1}-y_{2}\right)} \\
=-\left(\frac{x_{2}-x_{3}}{y_{2}-y_{3}}\right) x+\frac{x_{2}^{2}-x_{3}^{2}+y_{2}^{2}-y_{3}^{2}}{2\left(y_{2}-y_{3}\right)} \\
\Longrightarrow x\left(-\frac{x_{1}-x_{2}}{y_{1}-y_{2}}+\frac{x_{2}-x_{3}}{y_{2}-y_{3}}\right) \\
=\frac{x_{2}^{2}-x_{3}^{2}+y_{2}^{2}-y_{3}^{2}}{2\left(y_{2}-y_{3}\right)}-\frac{x_{1}^{2}-x_{2}^{2}+y_{1}^{2}-y_{2}^{2}}{2\left(y_{1}-y_{2}\right)} \\
\Longrightarrow x\left(\frac{\left(y_{1}-y_{2}\right)\left(x_{2}-x_{3}\right)-\left(y_{2}-y_{3}\right)\left(x_{1}-x_{2}\right)}{\left(y_{1}-y_{2}\right)\left(y_{2}-y_{3}\right)}\right) \\
=\frac{\left(y_{1}-y_{2}\right)\left(x_{2}^{2}-x_{3}^{2}+y_{2}^{2}-y_{3}^{2}\right)-\left(y_{2}-y_{3}\right)\left(x_{1}^{2}-x_{2}^{2}+y_{1}^{2}-y_{2}^{2}\right)}{2\left(y_{1}-y_{2}\right)\left(y_{2}-y_{3}\right)}
\end{gathered}
$$

Solving for $x$ we obtain

$$
x=\frac{\left(y_{1}-y_{2}\right)\left(x_{2}^{2}-x_{3}^{2}+y_{2}^{2}-y_{3}^{2}\right)-\left(y_{2}-y_{3}\right)\left(x_{1}^{2}-x_{2}^{2}+y_{1}^{2}-y_{2}^{2}\right)}{2\left[\left(y_{1}-y_{2}\right)\left(x_{2}-x_{3}\right)-\left(y_{2}-y_{3}\right)\left(x_{1}-x_{2}\right)\right]} .
$$

To obtain the $y$-coordinate of $C$, first, substitute the solution for $x$ into either bisector $\left(P_{1}, P_{2}\right)$ or bisector $\left(P_{2}, P_{3}\right)$, then:

$$
\begin{gathered}
y=\left(-\frac{x_{1}-x_{2}}{y_{1}-y_{2}}\right) . \\
\begin{array}{c}
\left(\frac{\left(y_{1}-y_{2}\right)\left(x_{2}^{2}-x_{3}^{2}+y_{2}^{2}-y_{3}^{2}\right)-\left(y_{2}-y_{3}\right)\left(x_{1}^{2}-x_{2}^{2}+y_{1}^{2}-y_{2}^{2}\right)}{2\left[\left(y_{1}-y_{2}\right)\left(x_{2}-x_{3}\right)-\left(y_{2}-y_{3}\right)\left(x_{1}-x_{2}\right)\right]}\right) \\
+\frac{x_{1}^{2}-x_{2}^{2}+y_{1}^{2}-y_{2}^{2}}{2\left(y_{1}-y_{2}\right)} .
\end{array}
\end{gathered}
$$


Simplifying this expression we obtain

$$
\begin{aligned}
& y= \\
& \frac{\left(x_{1}-x_{2}\right)\left(x_{2}^{2}-x_{3}^{2}+y_{2}^{2}-y_{3}^{2}\right)-\left(x_{2}-x_{3}\right)\left(x_{1}^{2}-x_{2}^{2}+y_{1}^{2}-y_{2}^{2}\right)}{2\left[\left(x_{1}-x_{2}\right)\left(y_{2}-y_{3}\right)-\left(x_{2}-x_{3}\right)\left(y_{1}-y_{2}\right)\right]} .
\end{aligned}
$$

It remains to be verified that this solution exists and is unique.

\section{$3.3 \quad$ Justifications}

Remark 3.1. We know our solution for $C$ exists if the denominators for both $x$ and $y$ are nonzero; namely, if $2\left[\left(y_{1}-y_{2}\right)\left(x_{2}-x_{3}\right)-\left(y_{2}-y_{3}\right)\left(x_{1}-x_{2}\right)\right] \neq 0$ and $2\left[\left(x_{1}-\right.\right.$ $\left.\left.x_{2}\right)\left(y_{2}-y_{3}\right)-\left(x_{2}-x_{3}\right)\left(y_{1}-y_{2}\right)\right] \neq 0$.

Consider the case that the denominator for $x$ is zero:

$$
\begin{aligned}
& 2\left[\left(y_{1}-y_{2}\right)\left(x_{2}-x_{3}\right)-\left(y_{2}-y_{3}\right)\left(x_{1}-x_{2}\right)\right]=0 \\
& \Longrightarrow\left(y_{1}-y_{2}\right)\left(x_{2}-x_{3}\right)-\left(y_{2}-y_{3}\right)\left(x_{1}-x_{2}\right)=0 \\
& \quad \text { (Dividing by 2) } \\
& \Longrightarrow\left(y_{1}-y_{2}\right)\left(x_{2}-x_{3}\right)=\left(y_{2}-y_{3}\right)\left(x_{1}-x_{2}\right) \\
& \Longrightarrow \frac{\left(y_{1}-y_{2}\right)}{\left(x_{1}-x_{2}\right)}=\frac{\left(y_{2}-y_{3}\right)}{\left(x_{2}-x_{3}\right)} \\
& \text { (Slopes of } \overleftrightarrow{P_{1} P_{2}} \text { and } \overleftrightarrow{P_{2} P_{3}} \text { are equal) }
\end{aligned}
$$

Similarly,

$$
\begin{aligned}
& 2\left[\left(x_{1}-x_{2}\right)\left(y_{2}-y_{3}\right)-\left(x_{2}-x_{3}\right)\left(y_{1}-y_{2}\right)\right]=0 \\
& \Longrightarrow\left(x_{1}-x_{2}\right)\left(y_{2}-y_{3}\right)-\left(x_{2}-x_{3}\right)\left(y_{1}-y_{2}\right)=0 \\
& \quad \text { (Dividing by } 2) \\
& \Longrightarrow \frac{\left(y_{2}-y_{3}\right)}{\left(x_{2}-x_{3}\right)}=\frac{\left(y_{1}-y_{2}\right)}{\left(x_{1}-x_{2}\right)} \\
& \text { (Slopes of } \overleftrightarrow{P_{1} P_{2}} \text { and } \overleftrightarrow{P_{2} P_{3}} \text { are equal) }
\end{aligned}
$$

We have shown that when the denominators of both expressions are zero, the slopes of ${\overleftrightarrow{P_{1} P_{2}}}_{1}$ and $\stackrel{P}{2}_{2} P_{3}$ are equal. Since both segments contain a common point $P_{2}$, this shows that $P_{1}, P_{2}$, and $P_{3}$ must be collinear, a contradiction of the assumptions of Theorem 1.14.

Because we divide by $\left(x_{1}-x_{2}\right)$ and $\left(x_{2}-x_{3}\right)$ above, we must demonstrate that these cannot be zero. Suppose that $x_{1}-x_{2}=0$. Then, $\left(y_{1}-y_{2}\right)\left(x_{2}-x_{3}\right)=\left(y_{2}-y_{3}\right)\left(x_{1}-\right.$ $\left.x_{2}\right) \Longrightarrow\left(y_{1}-y_{2}\right)\left(x_{2}-x_{3}\right)=0$. Thus, either $y_{1}-y_{2}=0$ or $x_{2}-x_{3}=0$. If $y_{1}-y_{2}=0$, then $y_{1}=y_{2}$. Since $x_{1}-x_{2}=0$ implies $x_{1}=x_{2}$, it follows that $P_{1}=P_{2}$, a contradiction. If $x_{2}-x_{3}=0$, then $x_{1}=x_{2}=x_{3}$, suggesting $P_{1}, P_{2}$, and $P_{3}$ are collinear, a contradiction.

Remark 3.2. The following verifies the uniqueness of our solution for $C$ :

We want to show that the point of intersection of two nonparallel lines exists and is unique. Suppose there exist two lines, $y=m_{1} x+b_{1}$ and $y=m_{2} x+b_{2}$, where $m_{1} \neq m_{2}$. We want to find the intersection of these two lines. Well, $m_{1} x+b_{1}=m_{2} x+b_{2} \Longrightarrow\left(m_{1}-m_{2}\right) x=b_{2}-b_{1}$. This gives a solution as long as the lines in question are not parallel to one another: $x=\frac{b_{2}-b_{1}}{m_{1}-m_{2}}$. By insert- ing this solution back into the equations for $x$, a solution for $y$ can be obtained: $y=m_{1}\left(\frac{b_{2}-b_{1}}{m_{1}-m_{2}}\right)+b_{1}$ and $y=m_{2}\left(\frac{b_{2}-b_{1}}{m_{1}-m_{2}}\right)+b_{2}$. Rewriting these expressions gives $y=\frac{m_{1} b_{2}-m_{1} b_{1}+m_{1} b_{1}-m_{2} b_{1}}{m_{1}-m_{2}}$ and $y=$ $\frac{m_{2} b_{2}-m_{2} b_{1}+m_{1} b_{2}-m_{2} b_{2}}{m_{1}-m_{2}}$, which reduce to the solution $y=\frac{m_{1} b_{2}-m_{2} b_{1}}{m_{1}-m_{2}}$. Thus, the intersection exists and is unique between two nonparallel lines.

\section{Conclusion}

We have proved directly that three noncolinear points, all of which are nonzero distance from each other, determine a unique circle of nonzero radius in $\mathbb{F}_{q}^{2}\left(q=p^{l}\right.$, $p>2$ is a prime, and $l \in \mathbb{N}$ ). Given three points which satisfy these conditions, it is possible to find the center of the circle they determine by finding the intersection of the perpendicular bisectors of two sets of the points. Using the definition of perpendicular bisector, it follows that this intersection is equidistant from all three points, showing that the intersection determines the center of a circle containing the three points. By the definition of a circle, deriving this center, showing it exists, and showing it is unique sufficiently demonstrates the existence of the circle containing the three points.

\section{Acknowledgments}

We would like to sincerely thank the referees for their careful review of our paper. Their comments significantly improved our paper and made it more rigorous. We would like to thank Dr. Joseph Stover for assistance with document editing.

\section{References}

[1] Axler, Sheldon. Linear Algebra Done Right. 2nd ed. Springer-Verlag New York, Inc. , 1997,1996. Print. 3

[2] Euclid. The Elements Book IV. Trans. Sir Thomas L. Heath. NewYork: Dover, 1956. Print.

[3] Gilbert, Jimmie, and Gilbert, Linda. Elements of Modern Algebra. 6th ed. Belmont: Thomson Books/Cole, 2005. Print.

[4] Lidl, Rudolf, and Niederreiter, Harald. Finite Fields. Cambridge University Press, 1997. Print. 